\section{Caracterización y determinación de ecotipos de oca (oxalis tuberosa), para el procesamiento de harinas en la Quebrada de Ancash, distrito y provincia de Yungay, Región Ancash.}

Recepción: Febrero de 2007 / Aceptación: Mayo de 2007
(1) Julio Yenque Dedios (2) Eulogio Santos De La Cruz (3) Julio Salas Bacalla (4) Osiris Feliciano Muñoz (5) Aurelio Lavado Soto

\section{RESUMEN}

El Perú tiene innumerables recursos naturales alimenticios de origen andino, uno de los cuales es la oca, la que aun no se ha aprovechado y masificado su consumo; razón por el que este estudio plantea hacer un análisis de los tipos de oca que existen en la sierra de la región Ancash, determinando sus características físicas y químicas para su posterior recomendación, dentro de un contexto de una posibilidad de industrialización. Para cumplir este cometido, han confluido esfuerzos profesionales y de campo. En este sentido ha sido posible la participación de la Universidad a través de sus laboratorios y Centros de Procesamiento, investigadores docentes e independientes y productores de oca, y con la finalidad de realizar ensayos de laboratorio para la obtención de harina y néctar.

Palabras Clave: Oca, néctar, harina de oca.

Characterization AND DeterminaTION OF OCA'S (OXALIS TUBEROSA) ECOTYPES, OR FLOUR PROCESSING IN THE ANCASH RAVINE, District AND PRovince OF YUNGAY, ANCASH REGION.

ABSTRACT

The country has innumerable nutritional natural resources of Andean character, one of which is oca, which not yet has taken advantage of and amassed its consumption; reason for which this study raises to make an analysis of the types of oca which they exist in the mountain range of the Ancash region, determining its physical and chemical characteristics for its later recommendation, dentroi of a context of an industrialization possibility. In order to fulfill this assignment, professional efforts and of field have come together. In this sense it has been possible the participation of the university through his laboratories and Centers of Processing, educational and independent, producing investigators of oca and population in general, with the purpose of making tests of laboratory for the obtaining of flour and nectar.

Key Words: Oca, nectar, flour of oca.

\section{INTRODUCCIÓN}

La oca, es un tubérculo andino, originario del sur del Perú, que se cultiva entre 3200 y 3900 m.s.n.m. contiene proteínas, carbohidratos y principalmente vitamina $\mathrm{C}$. Se consume mayormente sancochada, es muy dulce y agradable sobre todo si se deja solear. A fin de darle un valor agregado, se ha realizado un estudio preliminar con ensayos de laboratorio para producir harina y néctar, como resultado de las coordinaciones y trabajos de campo realizadas en la localidad de Shuyup Rayan (Yungay Ancash).

\section{INFORMACIÓN BÁSICA SOBRE EL TUBÉRCULO}

Originaria de los Andes Centrales, el origen de la oca podría estar entre el sur del Perú y Bolivia. Se cultiva en pequeñas parcelas asociadas a la papa, juntamente con la mashua y el olluco por ser parte de la dieta del agricultor y su familia (1).

Hábitat: La oca es un tubérculo que se cultiva entre 3200 a 3900 m.s.n.m.

Valor nutritivo: Contiene proteínas, carbohidratos y otros. La composición nutritiva en $100 \mathrm{gr}$. de la parte comestible del tubérculo fresco y de la kaya (tubérculo congelado y deshidratado) se presenta en el cuadro 1.

Cuadro 1. Composición nutritiva de la Oca (100 gr.)

\begin{tabular}{|l|c|c|}
\hline & Oca fresca & Kaya \\
\hline Energía (kcal) & 61 & 325 \\
Agua (\%) & 84.1 & 15.3 \\
Proteína (g) & 1 & 4.3 \\
Carbohidratos $(\mathrm{g})$ & 13.3 & 75.4 \\
Ceniza $(\mathrm{g})$ & 1 & 3.9 \\
Calcio (mg) & 2 & 52 \\
Fósforo (mg) & 36 & 171 \\
Hierro (mg) & 1.6 & 9.9 \\
Vitamina C (mg.) & 38.4 & 2.4 \\
\hline
\end{tabular}

Fuente: Tapia M. 1990. Cultivos andinos sub explotados y su aporte a la alimentación. Primera edición FAO (2).

(1) Ingeniero Industrial. Coordinador del Departamento de Diseño y Tecnología Industrial, UNMSM

E-mail: jyenqued@unmsmedu.pe

2) Magíster en Ciencias. Profesor del Departamento de Diseño y Tecnología Industrial, UNMSM.

E-mail: esantosd@unmsm edu pe

3) Ingeniero Industrial. Profesor del Departamento de Producción y Gestión Industrial, UNMSM

E-mail: jsalasb@unmsm.edu.pe

(4) Ingeniero Industrial. Profesor del Departamento de Producción y Gestión Industrial, UNMSM

E-mail: ofelicianom@unmsm.edu.pe

Ingeniero Químico. Profesor del Departamento de Diseño y Tecnología Industrial, UNMSM.

E-mail: mlavados@unmsm.edu.pe 
Usos: Se consume mayormente sancochada, es muy dulce y agradable sobre todo si se deja solear.

\section{PROCESAMIENTO PRELIMINAR}

El ensayo experimental a nivel de laboratorio, ha permitido obtener dos productos: harina y néctar, siendo este último producto el que podría constituir una realidad comercial en el corto plazo. El procesamiento básico se puede apreciar en los diagramas de flujo 1 y 2.
En el nivel del estudio realizado, el néctar es el producto de mayor interés, por lo que a manera de ilustración se presenta el balance de materia para dicho producto (3).

\section{Materias primas}

Las materias primas a utilizar son las siguientes:

- Oca

- Azúcar

- Aditivos

- Agua

\section{Diagrama 1. Obtención del néctar}
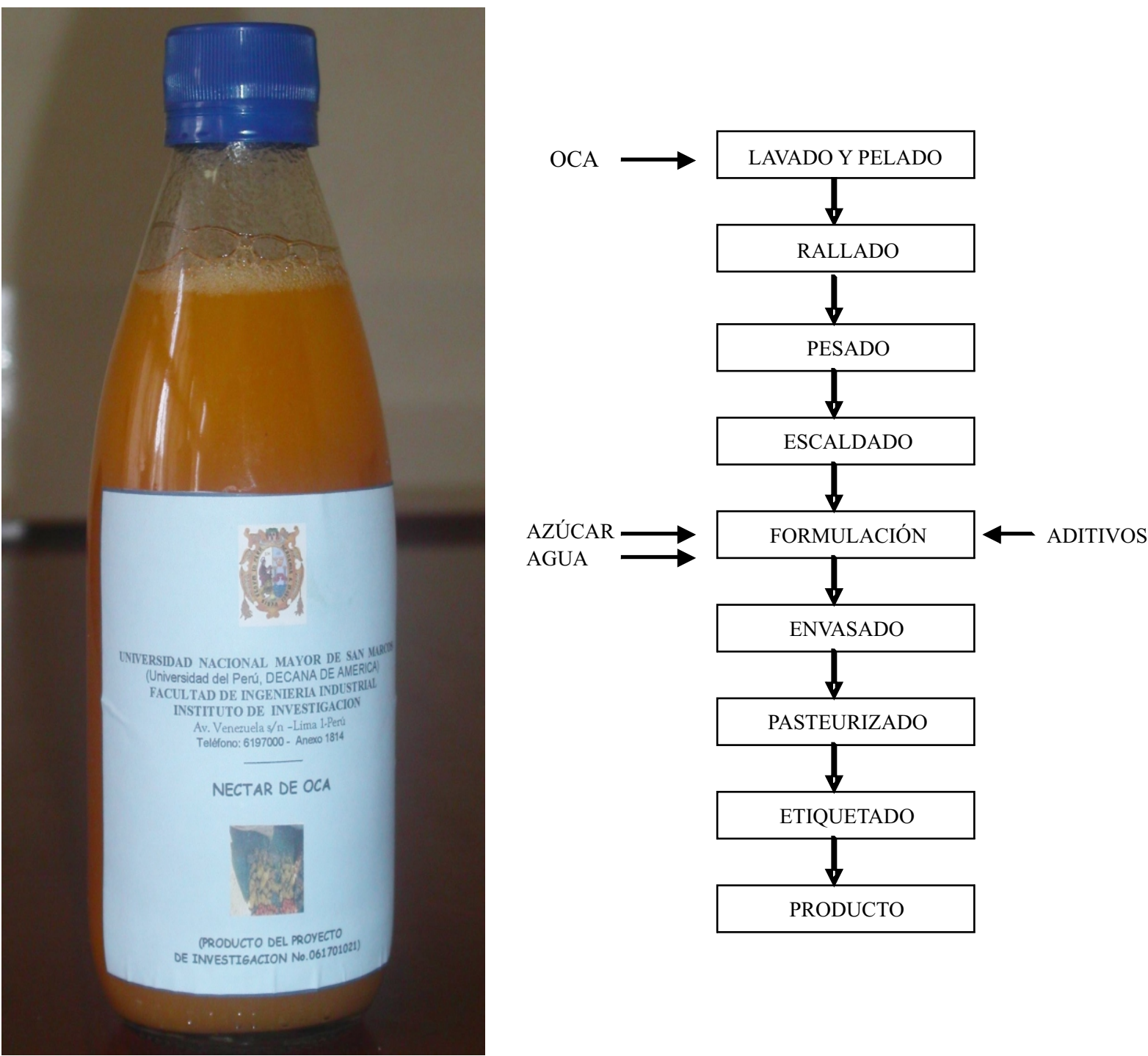


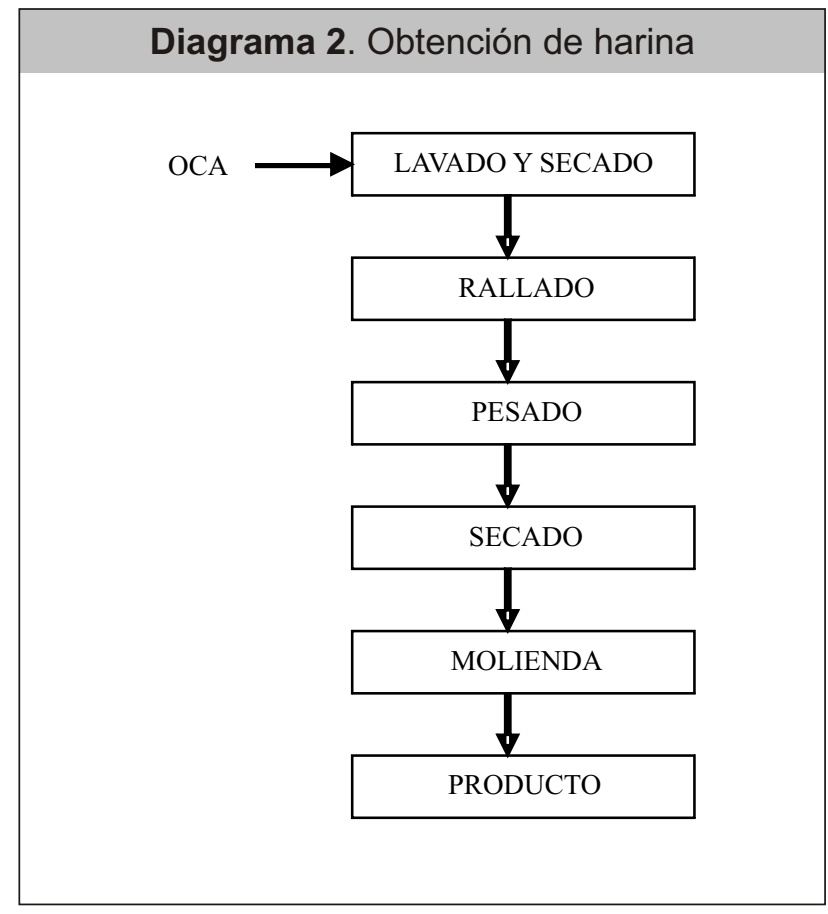

Parámetros

$\begin{array}{lll}\frac{\text { OCA }}{\text { AZÚCAR }} & =\frac{4}{3} \\ \frac{\text { OCA }}{\text { AGUA }}= & \frac{2}{9}\end{array}$

\section{Esquema del Proceso}

El balance de la materia se facilita o visualiza mejor, considerando el diagrama 3 , en el que se observa cuatro flujos de entrada y dos de salida. Como el período de escaldado es breve (15 minutos), la evaporación del agua es prácticamente nula. Los cálculos realizados permite conocer la concentración en masa de los sólidos presentes en el producto (néctar). El contenido de sólidos en la oca soleada se considera alrededor del $46 \%$ en masa. El total de aditivos se sitúa en un $2.4 \%$ respecto de la oca.

\section{Ecuaciones de balances de materia}

Balance Total

$\mathrm{M}_{1}+\mathrm{S}_{2}+\mathrm{L}_{3}+\mathrm{S}_{4}=\mathrm{mf}_{5}+\mathrm{G}_{6}$

Balance de Sólidos

$0,46 \mathrm{M}_{1}+\mathrm{S}_{2}+\mathrm{S}_{4}=\mathrm{mf}_{5} \cdot \mathrm{M}_{5}$

donde $\mathrm{mf}_{5}=$ fracción (composición) en masa de sólidos en el néctar.

\section{Diagrama 3. Balance de Materia}

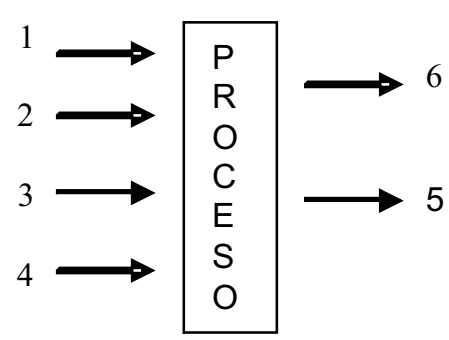

Identificación de los flujos o corrientes en el proceso

$$
\begin{aligned}
& 1=M_{1}=\text { Oca Pelada } \\
& 2=\mathrm{S}_{2}=\text { Azúcar } \\
& 3=\mathrm{L}_{3}=\text { Agua } \\
& 4=\mathrm{S}_{4}=\text { Aditivos } \\
& 5=\mathrm{mf}_{5}=\text { Producto (néctar) } \\
& 6=\mathrm{G}_{6}=\text { Vapor de Agua }
\end{aligned}
$$

Balance de Agua

$0,54 \mathrm{M}_{1}+\mathrm{L}_{3}=\mathrm{G}_{6}+\left(1-\mathrm{mf}_{5}\right) \cdot \mathrm{M}_{5}$

donde $\left(1 . \mathrm{mf}_{5}\right)=$ fracción en masa de líquido (agua) en el néctar.

\section{CÁLCULOS}

Base de cálculos: $100 \mathrm{~kg}$. de oca pelada $=M_{1}$

\section{De acuerdo a los parámetros:}

$$
\begin{aligned}
& \mathrm{S}_{2}=\frac{3}{4} \times 100=75 \mathrm{Kg} \\
& \mathrm{L}_{3}=\frac{9}{2} \times 100=450 \mathrm{Kg}
\end{aligned}
$$

$S_{4}=0,024 \times 100=2,4$

Reemplazando en la ecuación (1) se tiene, $100+75+450+2,4=M_{5}+G_{6}$

Debido a que la evaporación del agua durante el escaldado, es prácticamente despreciable, G6 $=0$.

\section{En consecuencia,}

$M_{5}=627,4 \mathrm{~kg}$.

Recurriendo a la ecuación (3) se obtiene: $\mathrm{mf}_{5} \cdot 0,54 \times 100+450=0+\left(1-\mathrm{mf}_{5}\right) \times 627,4$ $\mathrm{mf}_{5}=0,20$ 


\section{RESULTADO DE LOS CÁLCULOS:}

El néctar o producto obtenido contiene, de acuerdo a los cálculos realizados el $20 \%$ de sólidos en masa.

\section{CONCLUSIONES Y RECOMENDACIONES}

El primer ensayo realizado a nivel de laboratorio debe ser continuado con pruebas en Planta Piloto. La continuación de los estudios debe considerar la formulación de otros derivados de la oca, tales como confitados y alcohol.

Así como la incorporación de nuevas áreas geográficas de cultivo y una gran gama de variedades o ecotipos. Debe contemplarse la posibilidad de formulaciones con otros productos naturales como el camu camu, por su gran contenido de vitamina $\mathrm{C}$ y edulcorantes nativos.
La posibilidad de industrialización de la oca amerita ampliar los estudios hacia otras variedades afines como la mashua, de la que se tiene información acerca de sus propiedades terapéuticas y curativas relacionadas con la próstata.

\section{REFERENCIAS BIBLIOGRÁFICAS}

1. Fairlie,T. Morales, M. Holle (1999). Raíces y Tubérculos Andinos. CIP, Centro Internacional de la Papa. Lima, Perú.

2. Garcia G., Quintero R., Lopez M., (2005) Biotenologia Alimentaria.

3. INIA, Instituto Nacional de Investigaciones Agrarias (2006). Hojas Divulgativas de los Cultivos Nativos del Perú. Segunda Edición. Lima, Perú. 